\title{
BIAXIAL STRAIN EFFECTS ON OPTICAL CHARACTERISTICS OF QUANTUM WELL LASERS
}

\author{
J. S. HSU, MING RONG LEE and K. F. YARN* \\ Far East College, Department of Electrical Engineering, \\ Optoelectronic Semiconductor Center, Hsin-Shih, Tainan, \\ Taiwan 744, Republic of China
}

(Received 30 June 2000; In final form 12 September 2000)

\begin{abstract}
The effects of biaxial strain produced by the lattice mismatch of constituent materials on the optical properties of strained $I n_{1-x} G a_{x} A s_{y} P_{1-y} / I n_{1-x} G a_{x} A s$ quantum well lasers are investigated.

The optical gain and refractive index change of a biaxially stressed quantum well lasers are studied theoretically using the multiband effective mass equation (i.e., $\vec{k} \cdot \vec{p}$ method), deformation potential theory and Fermi-Golden rule, band mixing effect is retained in the calculations. It is found that the biaxial strain would change the subband structures and optical properties of quantum well lasers, we found the gain of TE mode increases with increasing compressive strain, while the gain of TM mode increases with increasing tensile strain, these will be benefited for reducing the threshold current depending on whether the quantum well lasers are operating in TE or TM mode. On the other hand, the refractive index change in the active region near the TE(TM) mode peak gain becomes more negative when a biaxial compressive(tensile) strain is applied, it leads to the conclusion that the strain weakens the optical confinement, the temperature dependence of gain also becomes stronger when there is strain.

Finally, we also found the minimum peak gain occurs when a small tensile strain is applied, but no strain.
\end{abstract}

Keywords: Biaxial strain; Lattice mismatch; Refractive index; Tensile strain

\section{INTRODUCTION}

When the lattice mismatch of constituent materials is greater than $0.1 \%$, we called such semi-conductor materials are lattice-mismatched.

*Address for correspondence: P.O. Box 345, Tainan, Taiwan 704, Republic of China. 
Previous heterostructure work is primarily focused on lattice-matched system due to the degradation of electronic and optical properties of devices made from lattice-mismatched material systems. Since the advance in epitaxial growth, it becomes possible to obtain a latticemismatched heterostructure without dislocation generated at interface if the thickness of constituent materials are thin enough, such high quality heterostructure called as "strained-layer structure", such as InGaAs/GaAs, InP/InGaAs... etc. Recently, these strained layer structures have wide applications in lasers [1 -4], photodetector [5] and transistor $[6,7]$, due to their flexibilities in the choice of mismatched materials.

Previously, the theoretical studies are focused on uniaxially stressed quantum wells [8-10], there will be valuable to realize the electronic and optical properties of strained-layer quantum wells, but unrealistic in device applications. In this work, the effects of the biaxial strain produced by the lattice mismatched of constituent materials on the optical gain in quantum well lasers are investigated, the refractive index change also be calculated in order to understand the guiding behavior of laser light in the active region.

Since the optical gain and refractive index change are closely related to the band structure of the semiconductor, we first calculated the band structures of strained quantum wells using the multiband effective mass equation and introducing the strain by the deformation potential theory. Once the subband structures and wave functions are obtained, the gain and refractive index change can be calculated based on Fermi-Golden rule [11] with the broadening effect taken into account.

\section{THEORY}

Considering a strained-layer quantum well grown on [001] direction. Since all lasers properties are closely related to the band structure of semiconductor, first, we use multiband effective mass equation (i.e., $\vec{k} \cdot \vec{p}$ method) $[12,13]$ incorporating with deformation potential theory to calculate both the subband structures and wavefunctions of strained-layer quantum well. For simplicity, we assume that the conduction and valence bands are decoupled, in fact, which is a good 
approximation for wide bandgap semiconductor. For the conduction band, a simple parabolic approximation is used, while using Luttinger-Kohn Hamiltonian to describe the valence bands. In order to simplify the calculations, we make a unitary transformation of a $4 \times 4$ Hamiltonian with a biaxial strain into a block-diagonal form:

$$
H_{t}=-\left[\begin{array}{cccc}
P+Q+\zeta & \tilde{R} & 0 & 0 \\
\tilde{R}^{\dagger} & P-Q-\zeta & 0 & 0 \\
0 & 0 & P-Q-\zeta & \tilde{R} \\
0 & 0 & \tilde{R}^{\dagger} & P+Q+\zeta
\end{array}\right]+V_{h}(z)
$$

where

$$
\begin{aligned}
& P=\left(\frac{\hbar^{2}}{2 m_{o}}\right) \gamma_{1}\left(k_{x}^{2}+k_{y}^{2}-\frac{\partial^{2}}{\partial z^{2}}\right) \\
& Q=\left(\frac{\hbar^{2}}{2 m_{o}}\right) \gamma_{2}\left(k_{x}^{2}+k_{y}^{2}+2 \frac{\partial^{2}}{\partial z^{2}}\right) \\
& \tilde{R}=\left(\frac{\hbar^{2}}{2 m_{o}}\right)\left[\sqrt{3} \bar{\gamma}\left(k_{x}^{2}+k_{y}^{2}\right)-2 \sqrt{3} \gamma_{3}\left(k_{x}^{2}+k_{y}^{2}\right)^{1 / 2} \frac{\partial}{\partial z}\right]
\end{aligned}
$$

here, $V_{h}(z)$ is the potential profile of valence band; $\gamma_{1}, \gamma_{2}, \gamma_{3}$ are Luttinger parameters; $m_{o}$ is the free electron mass; $\bar{\gamma}=1 / 2\left(\gamma_{1}+\gamma_{2}\right)$, $\tilde{R}^{\dagger}$ is the adjoint of $\tilde{R}$, and deformation potential $\zeta$ define as follow [15]:

$$
\zeta=-b\left(1+\frac{2 C_{12}}{C_{11}}\right) \varepsilon
$$

where $b$ is the deformation potential, $C_{11}$ and $C_{12}$ are elastic constant $\left(\right.$ dyne $\left./ \mathrm{cm}^{2}\right)$, and $\varepsilon$ is strain define as below:

$$
\varepsilon=\frac{a_{o}-a(x)}{a_{o}}
$$

where

$a_{0} \quad$ in-plane lattice constant.

$a(x)$ lattice constant of quantum well. 
Now, the $4 \times 4$ Hamiltonian are decoupled, the upper and lower block wave functions have the form:

$$
\begin{aligned}
& \Psi_{m \vec{k} \|}^{U}(\vec{r})=\sum_{\nu=1,2} g_{m}^{(\nu)}\left(\vec{k}_{\|}, z\right) e^{i \vec{k}_{\|} \cdot \vec{p}}|\nu\rangle \\
& \Psi_{m \vec{k} \|}^{L}(\vec{r})=\sum_{\nu=3,4} g_{m}^{(\nu)}\left(\vec{k}_{\|}, z\right) e^{i \vec{k}_{\|} \cdot \vec{p}}|\nu\rangle
\end{aligned}
$$

where $g_{m}^{(\nu)}\left(\vec{k}_{\|}\right)$are the envelope functions, $\{|\nu\rangle\}$ denote the transformed Bloch functions at the zone center, $|\nu\rangle$ refers to new basis [10], $m$ is the subband index, $\vec{k}_{\|}=k_{x} \hat{x}+k_{y} \hat{y}, \vec{\rho}=x \hat{x}+y \hat{y}, U$ and $L$ are associated with upper and lower block Hamiltonians $H^{U}$ and $H^{L}$, and

$$
H^{\sigma}=\left[\begin{array}{cc}
P \pm Q \pm \zeta & \tilde{R} \\
\tilde{R}^{\dagger} & P \mp Q \mp \zeta
\end{array}\right], \quad \sigma=U \text { or } L
$$

We solve the coupled eigenvalue problems numerically given by (7) using the finite difference method. Once the subband levels and wavefunctions are known, the optical gain $g(\omega)$ and refractive index change $\left(\Delta n_{r} / n_{r}\right)$ derived based on Fermi-Golden rule with broadening effect taken into account are:

$$
\begin{aligned}
g(\omega)= & \frac{\pi e \mu_{o} c}{n_{r} m_{o} \omega V} \sum_{\sigma \eta} \sum_{l m} \sum_{\vec{k}_{\|}}\left[f_{c}^{l}\left(\vec{k}_{\|}\right)-f_{v}^{m}\left(\vec{k}_{\|}\right)\right]\left|\hat{\varepsilon} \cdot \vec{M}_{\sigma \eta}^{l m}\left(\vec{k}_{\|}\right)\right| \\
& \left\{\frac{\Gamma / \pi}{\left[E_{c v}^{l m}\left(\vec{k}_{\|}\right)-\hbar \omega\right]+\Gamma}\right\} \\
\frac{\Delta n_{r}}{n_{r}}= & \frac{\pi e \mu_{o} c}{2 n_{r} m_{o} \omega V} \sum_{\sigma \eta} \sum_{l m} \sum_{\vec{k}_{\|}}\left[f_{c}^{l}\left(\vec{k}_{\|}\right)-f_{v}^{m}\left(\vec{k}_{\|}\right)\right]\left|\hat{\varepsilon} \cdot \vec{M}_{\sigma \eta}^{l m}\left(\vec{k}_{\|}\right)\right| \\
& \left\{\frac{1}{\pi} \frac{E_{c v}^{l m}\left(\vec{k}_{\|}\right)}{\left[E_{c v}^{l m}\left(\vec{k}_{\|}\right)-\hbar \omega\right]+\Gamma}\right\}
\end{aligned}
$$

where $\sigma$ denotes the upper and lower blocks of Hamiltonian, $\eta$ is the electron spin state, $\hat{\varepsilon}$ is the polarization vector of optical field, $\vec{M}_{\sigma \eta}^{l m}\left(\vec{k}_{\|}\right)$ is the optical matrix element between the $l$ th subband in the conduction band with spin state $\eta$ and $m$ th subband in the valence band of the $2 \times 2$ Hamiltonian $H^{\sigma}, \omega$ is the angular frequency, $f_{c}^{l}, f_{v}^{m}$ 
are distribution functions, $E_{c v}^{l m}\left(\vec{k}_{\|}\right)$is the transition energy between $l$ th subband in the conduction band and $m$ th subband in the valence band, $\Gamma$ is the broadening parameter. In multiband effective mass approximation, the optical matrix element is given by [16]

$$
\hat{\varepsilon} \cdot \vec{M}_{\sigma \eta}^{l m}\left(\vec{k}_{\|}\right)= \begin{cases}\sum_{\nu=1,2}\left\langle g_{m}^{(\nu)} \mid \phi_{l}\right\rangle\langle\nu|\hat{\varepsilon} \cdot \vec{P}| S, \eta\rangle, & \sigma=U \\ \sum_{\nu=3,4}\left\langle g_{m}^{(\nu)} \mid \phi_{l}\right\rangle\langle\nu|\hat{\varepsilon} \cdot \vec{P}| S, \eta\rangle, & \sigma=L\end{cases}
$$

where

$\phi_{l}$ the envelope function for electron.

$S$ the periodic part of Block function at zone center for electrons.

\section{RESULTS AND DISCUSSIONS}

Here, we take biaxially stressed $I n_{1-x} G a_{x} A s$ grown on an $I n_{1-x} G a_{x}$ $A s_{y} P_{1-y}$ lattice matched to InP and having barrier bandgap $E_{g}=0.95$ $e v$ as a numerical example. For $G a$ mole fraction $x=0.47$, the system is lattice matched, while the quantum well will suffer a biaxial compressive strain for $x<0.47$, and under a biaxial tension condition for $x>0.47$.

In Figures 1(a) and (c), we show the valence band structure along the $k_{x}$ direction for (a) lattice matched $(x=0.47)$, (b) compressive $(x<0.47)$, (c) tensile $(x>0.47)$ conditions. In Figure 1(a), we could found that the mixing of light (heavy) hole state into heavy(light) hole state would give rise to strong nonparabolicities in the valence band structure for $\vec{k}_{\|} \neq 0$. By the way, the VB1 (first valence band) is heavy hole band, while VB2 is light hole band for lattice matched condition. In Figure 1(b), the first four subband of valence band are calculated under a biaxial compression. It is interesting to note that the LH1 is pushed down well below HH1, and the highly nonparabolic HH2 becomes parabolic in a wide range of $k_{\|}$values, thus the admixture of light hole state into heavy hole state would decrease. Figure 1(c) show the subband structure under the tension, we found that the LH1 band has been pushed up above the HH1 band, this crossing of HH1 and LH1 would change the laser light polarization from TE mode to TM mode as can be seen later. 

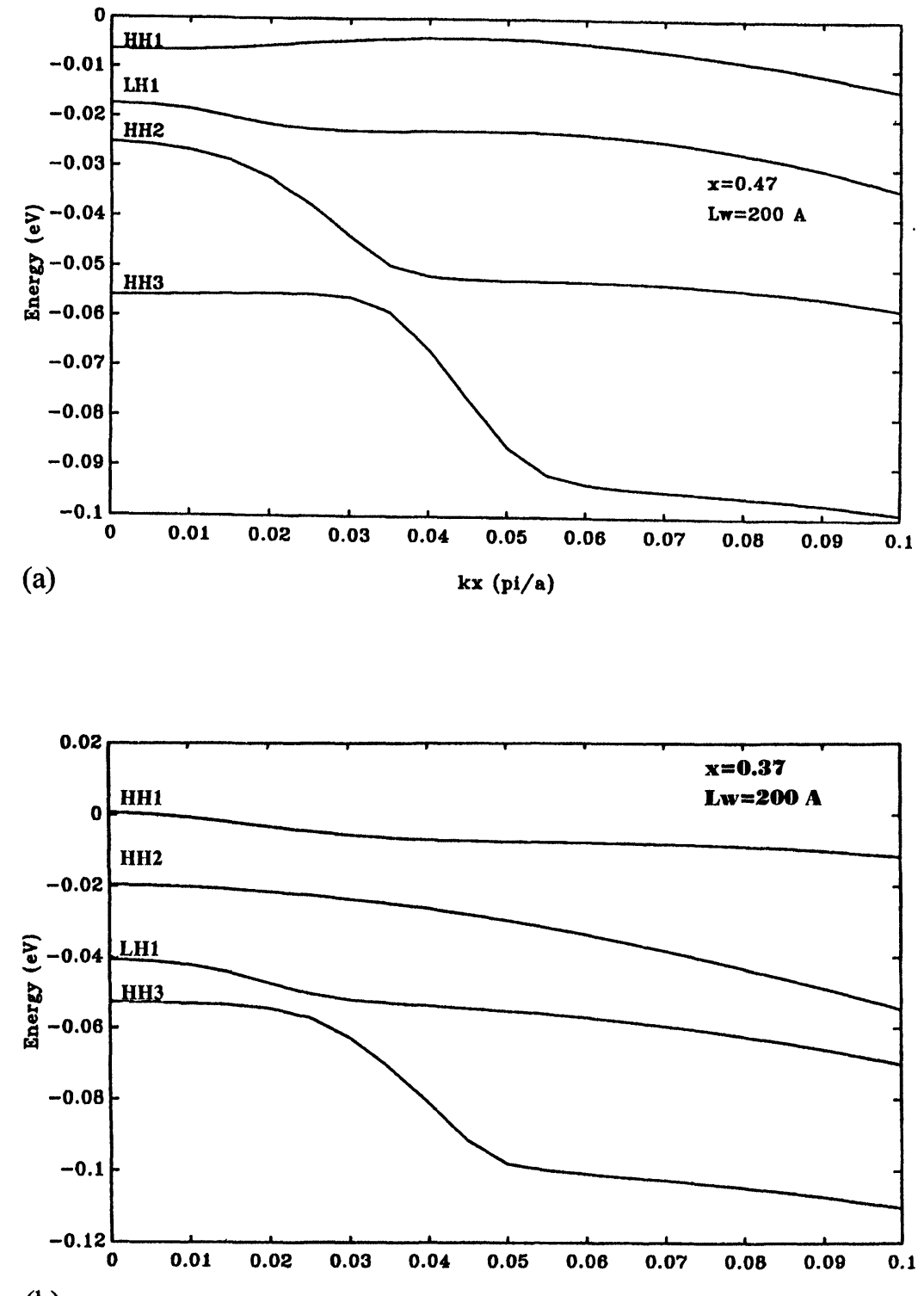

(b)

$\mathbf{k x}(\mathrm{pi} / \mathrm{a})$

FIGURE 1 (a) The valence band structure for lattice matched $(x=0.47)$ with well width $\mathrm{Lw}=200 \AA$. (b) The valence band structure for compression $(x=0.37)$ with well width $\mathrm{Lw}=200 \AA$. (c) The valence band structure for tension $(x=0.57)$ with well width $\mathrm{Lw}=200 \AA$. 


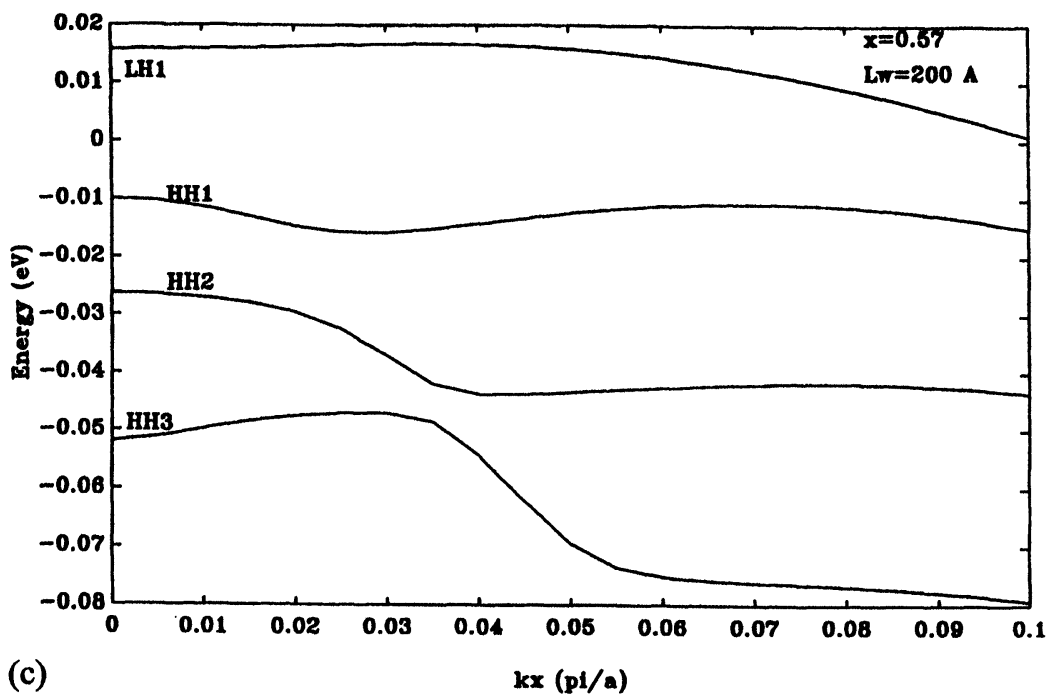

FIGURE 1 (Continued).

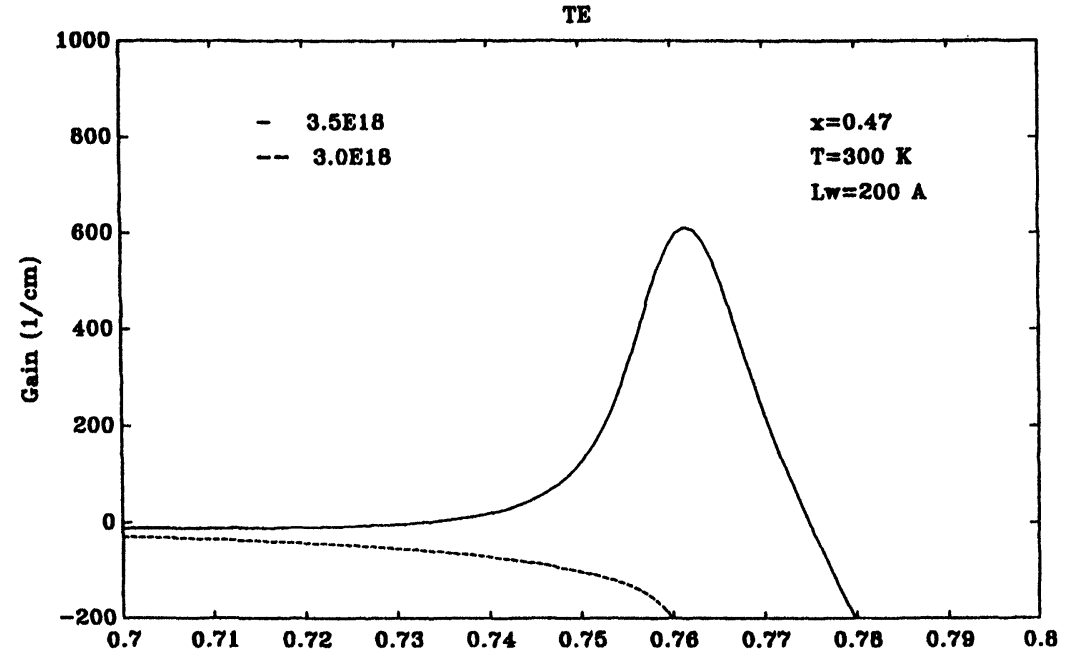

(a)

Photon energy (ev)

FIGURE 2 The TE mode gain spectra for $200 \AA$ under (a) lattice-matched, (b) compression, (c)tension. 


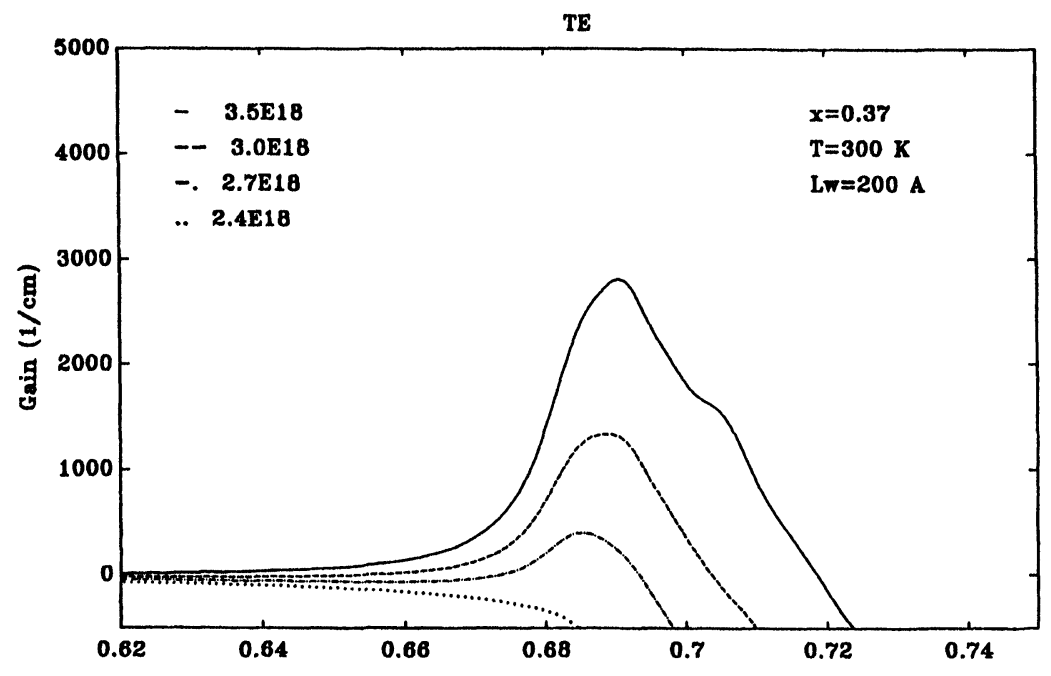

(b)

Photon energy (eV)

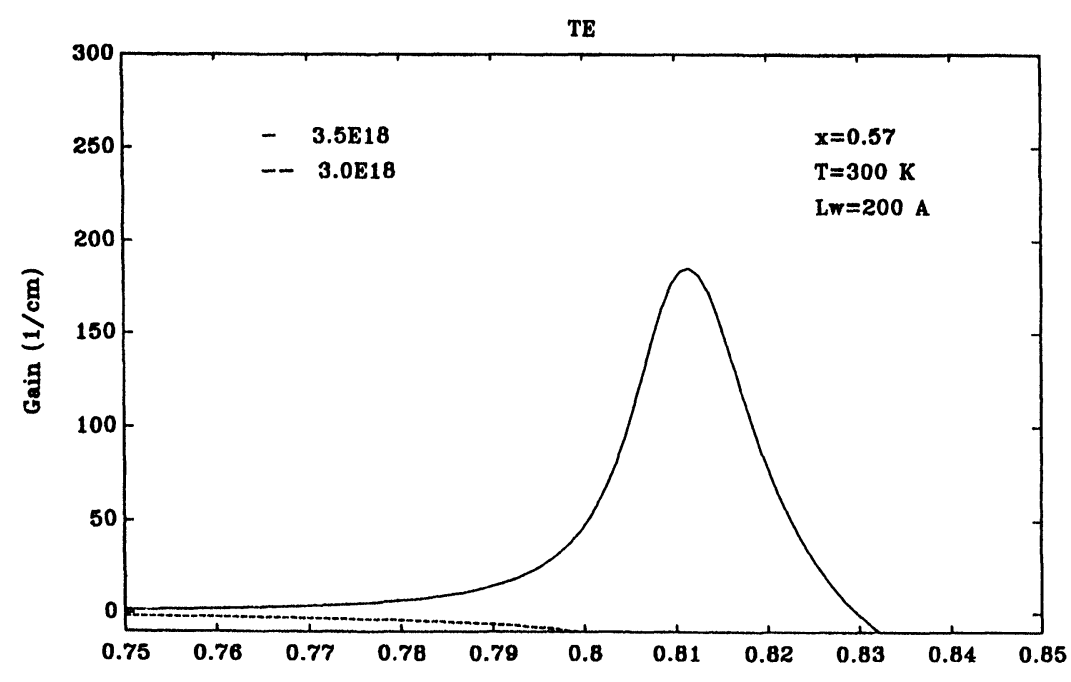

(c)

Photon energy (ev)

FIGURE 2 (Continued). 
The gain spectra of TE mode (optical electric field polarized along $x$ direction) at $300 \mathrm{~K}$ are shown in Figure 2 for lattice-matched (Fig. 2(a)), compressive (Fig. 2(b)) and tensile (Fig. 2(c)) condition for various carrier densities with well width $200 \AA$. It is interesting to see a quite enhancement of TE mode gain when there is a biaxial compressive strain. This is because of (i) the decrease of admixture of light hole state into heavy hole state under compression, (ii) the TE mode optical matrix element would increase near the zone center with compressive strain, and (iii) for a given carrier densities, it is more easy to populate inversion for compressive condition. In contrast, Figure 3 show the TM mode (optical electric field polarized along $\mathrm{z}$ direction) gain under lattice-matched (Fig. 3(a)), compression (Fig. 3(b)) and tension (Fig. 3(c)). We found the TM polarization would be the dominant laser light when suffering a tensile strain.

We also calculated the refractive index change for TE and TM mode in Figures 4 and 5 respectively. Figure 4 (Fig. 5) shown that the refractive index change for TE(TM) polarization in the active region near the peak gain become more negative when a biaxial

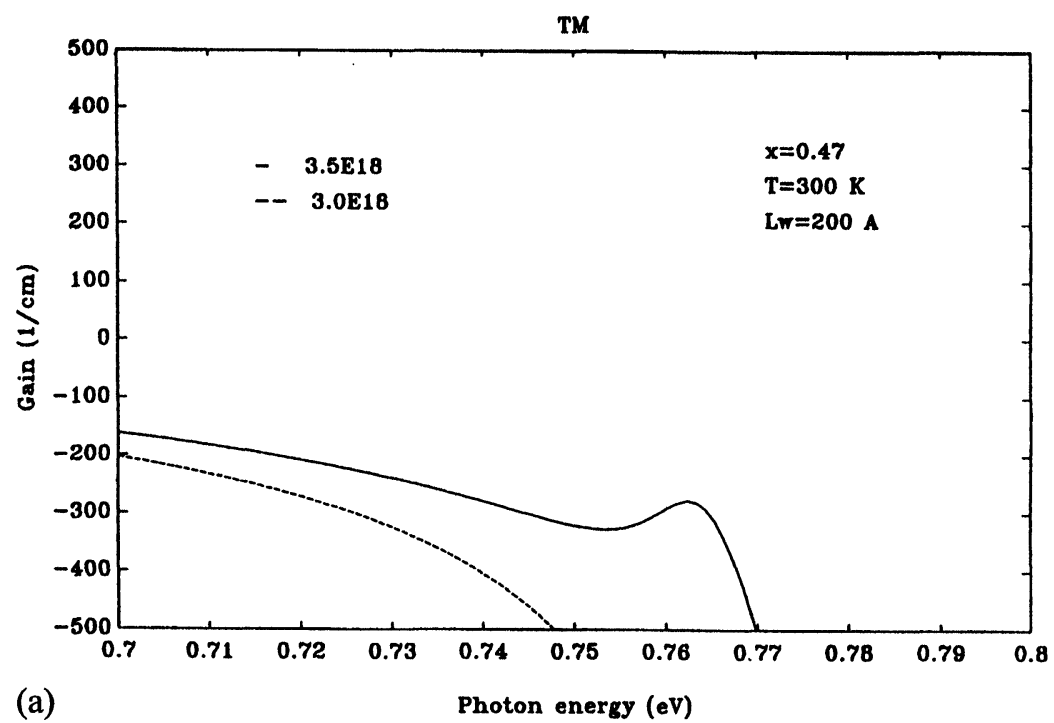

FIGURE 3 The TM mode gain spectra for $200 \AA$ under (a) lattice-matched, (b) compression, (c) tension. 


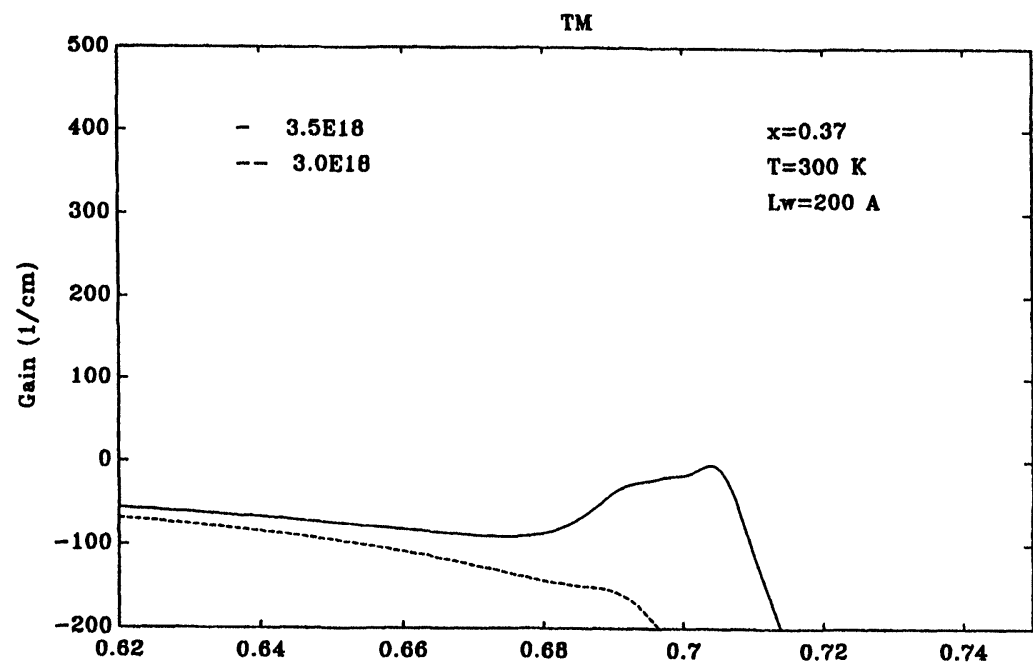

(b)

Photon energy (ev)

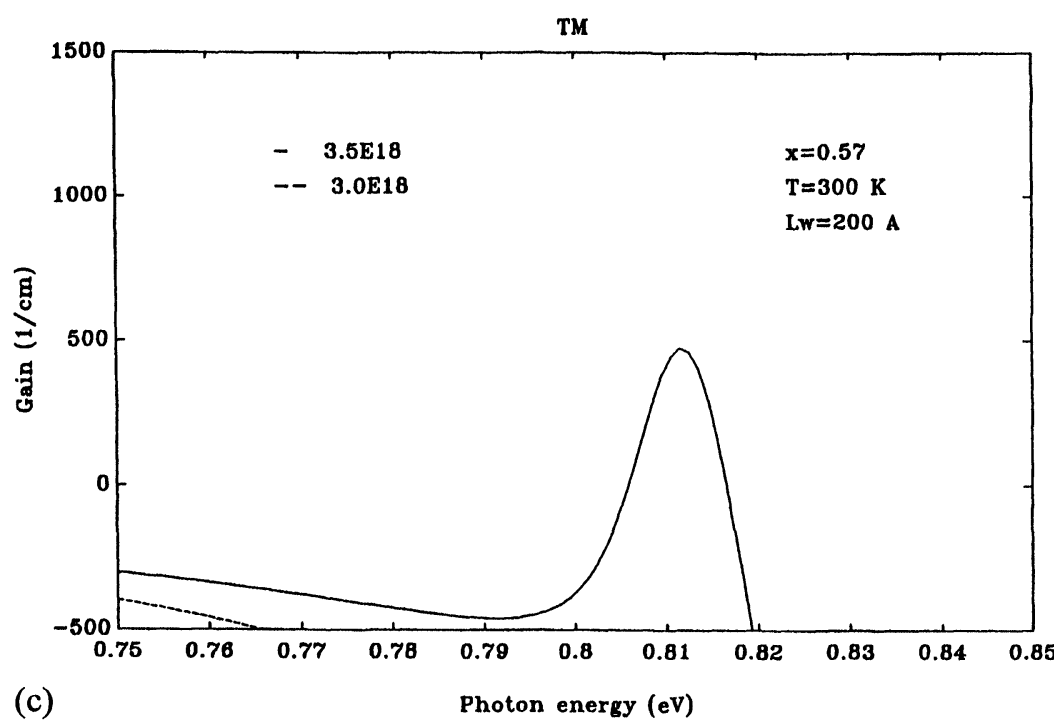

FIGURE 3 (Continued) 

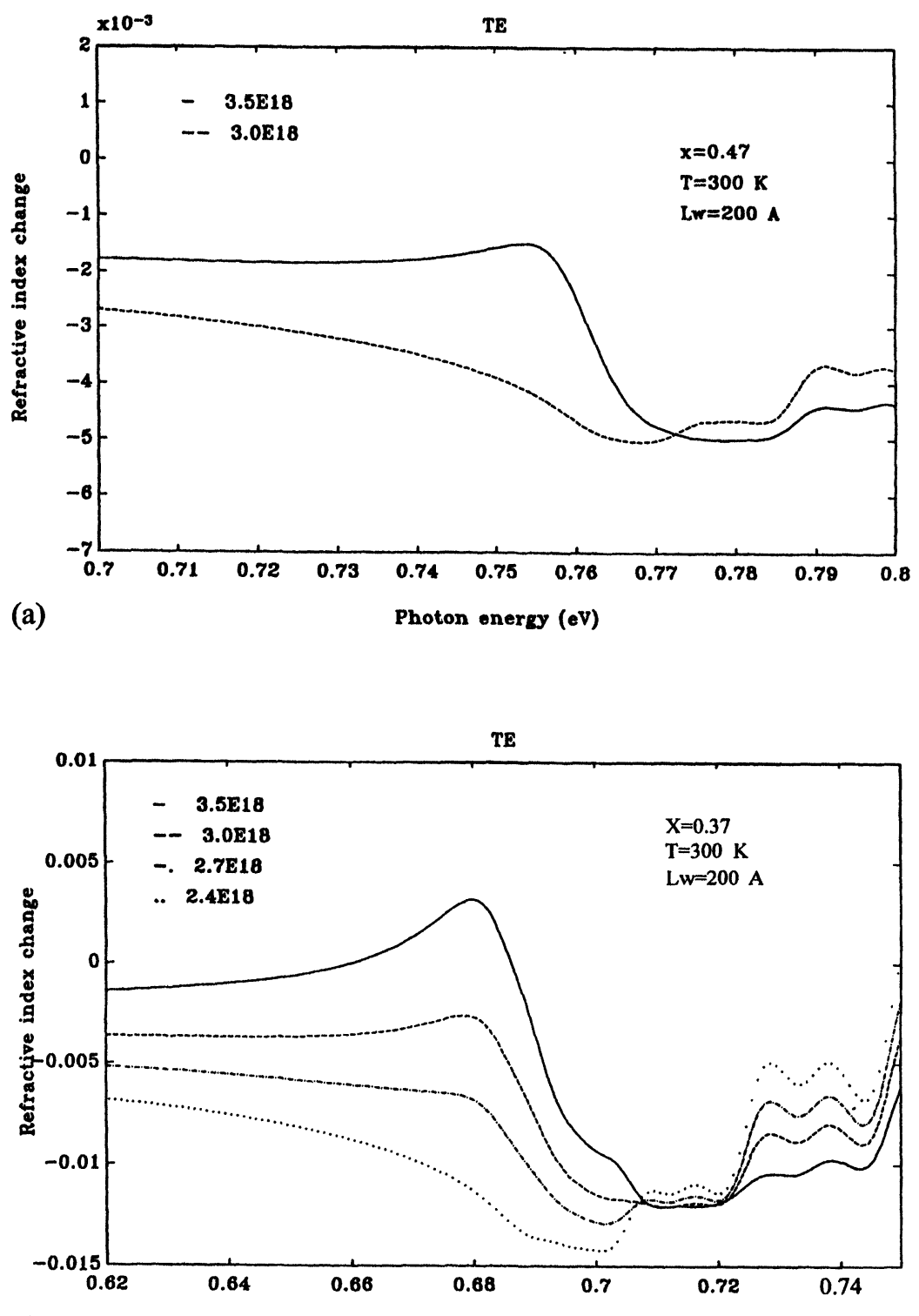

(b)

Photon energy (ev)

FIGURE 4 The TE mode refractive index change for $200 \AA$ (a) lattice-matched, (b) compression, (c) tension. 


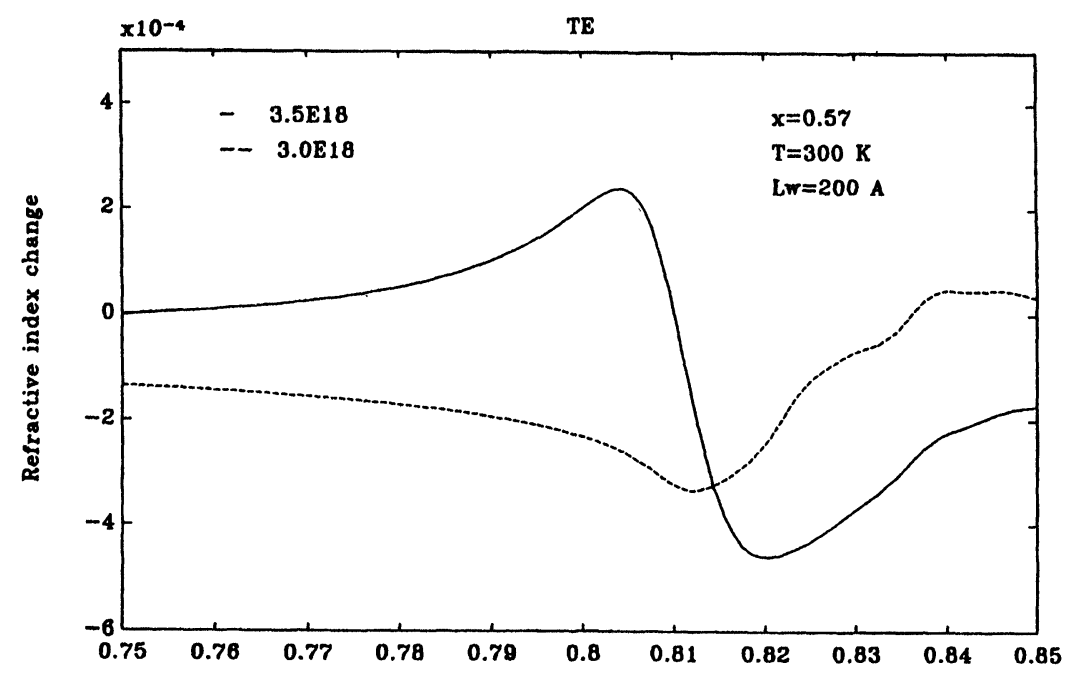

(c)

Photon energy (ev)

FIGURE 4 (Continued).

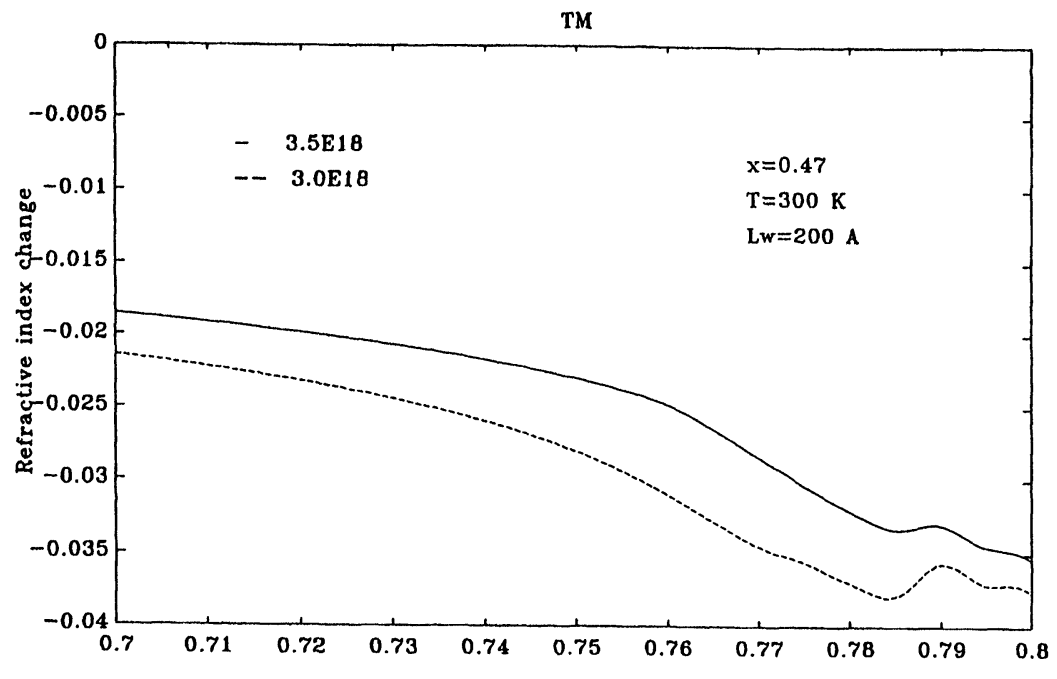

(a)

Photon energy (eV)

FIGURE 5 The TM mode refractive index change for $200 \AA$ (a) lattice-matched, (b) compression, (c) tension. 


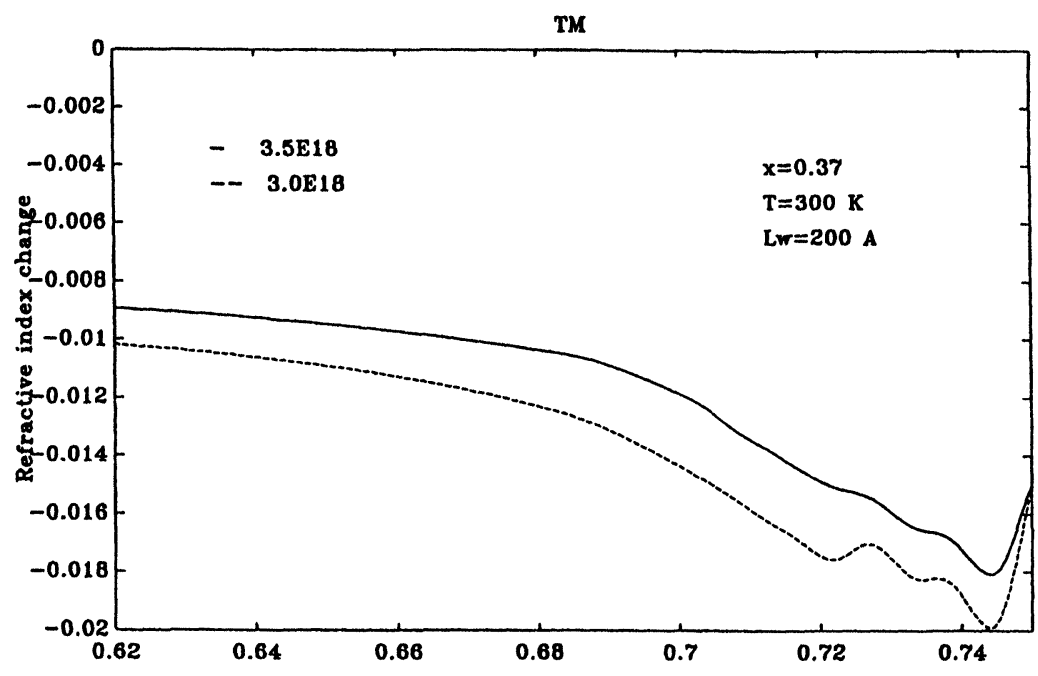

(b)

Photon energy (ev)

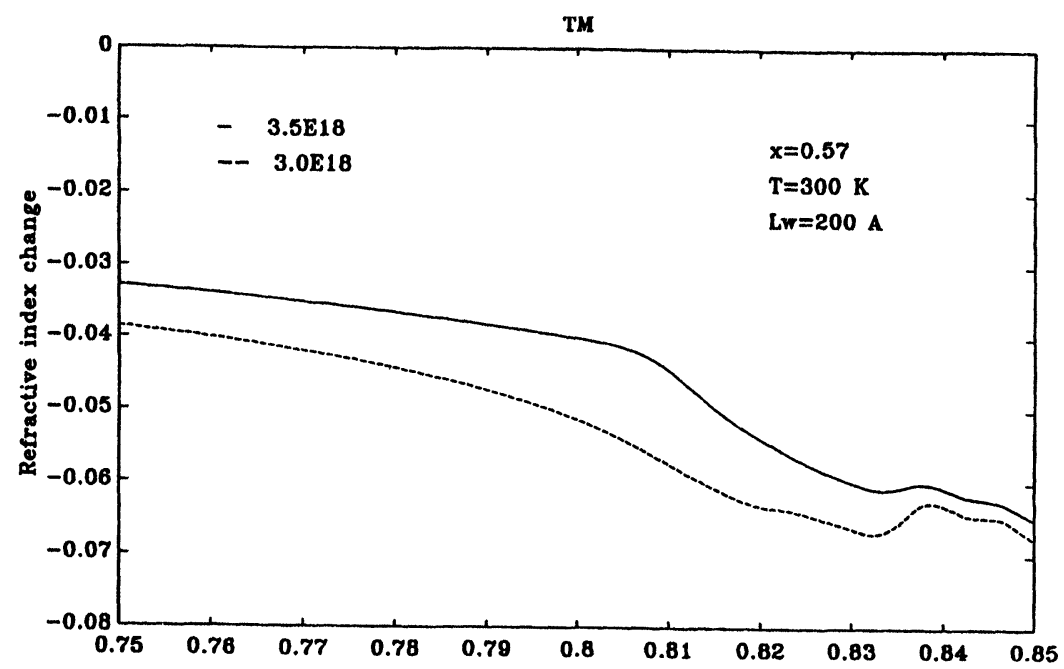

(c)

Photon energy (ev)

FIGURE 5 (Continued). 
compressive(tensile) strain is applied. In other words, the biaxial compressive (tensile) strain weakened the optical confinement of TE(TM) mode laser light.

The temperature dependence of gain is one of the most important parameters of laser diodes during operation. Here, we show the temperature dependence of TE and TM mode gain in Figure 6. In Figure 6(a), we found that the TE mode gain would decrease more rapidly with increasing temperature when there is a biaxial compressive strain, while the TM mode gain would decrease faster with increasing temperature by applying a biaxial tensile strain (Fig. 6(b)). It is interesting to found that the TM mode gain under tension would raise more rapidly than TE mode gain under compression with decreasing temperature. The TM mode gain reached up to $15000 \mathrm{~cm}^{-1}$ under tension at $100 \mathrm{~K}$ !

Finally, we calculated the peak gain for various strain, and found the minimum peak gain of both TE (Fig. 7(a)) and TM (Fig. 7(b)) mode occurred when a small tensile strain $(x \simeq 0.51)$ is applied, but no strain. This is primarily because that the LH1 band is still under HH1 band under small tension.

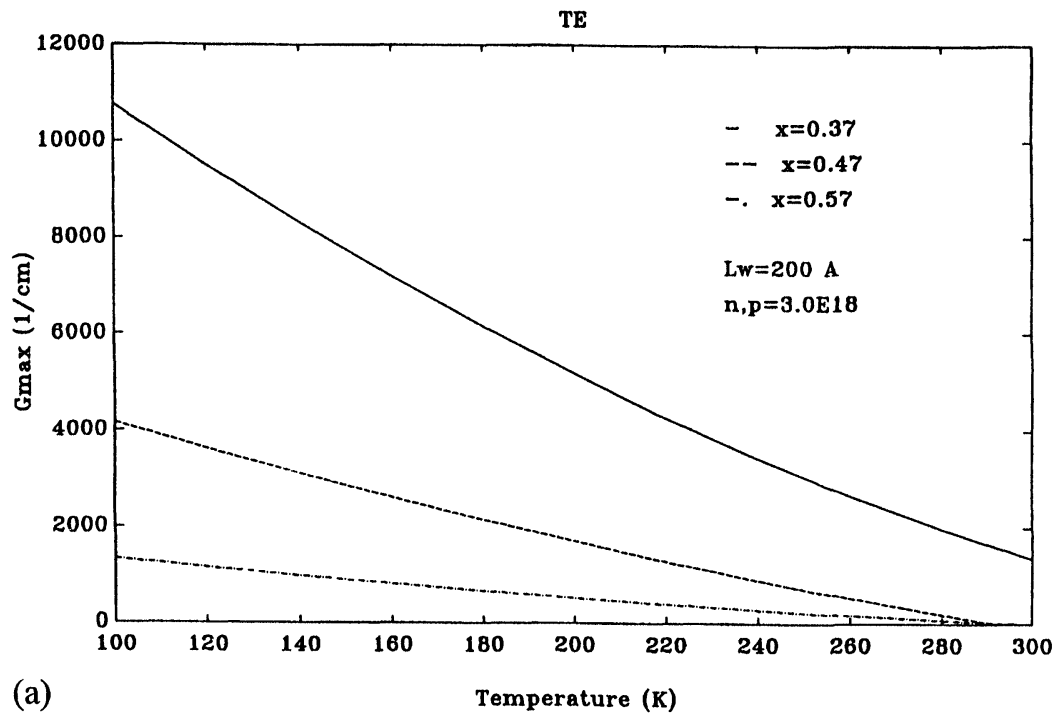

FIGURE 6 The temperature dependence of (a) TE, (b) TM mode peak gain for different strain. 


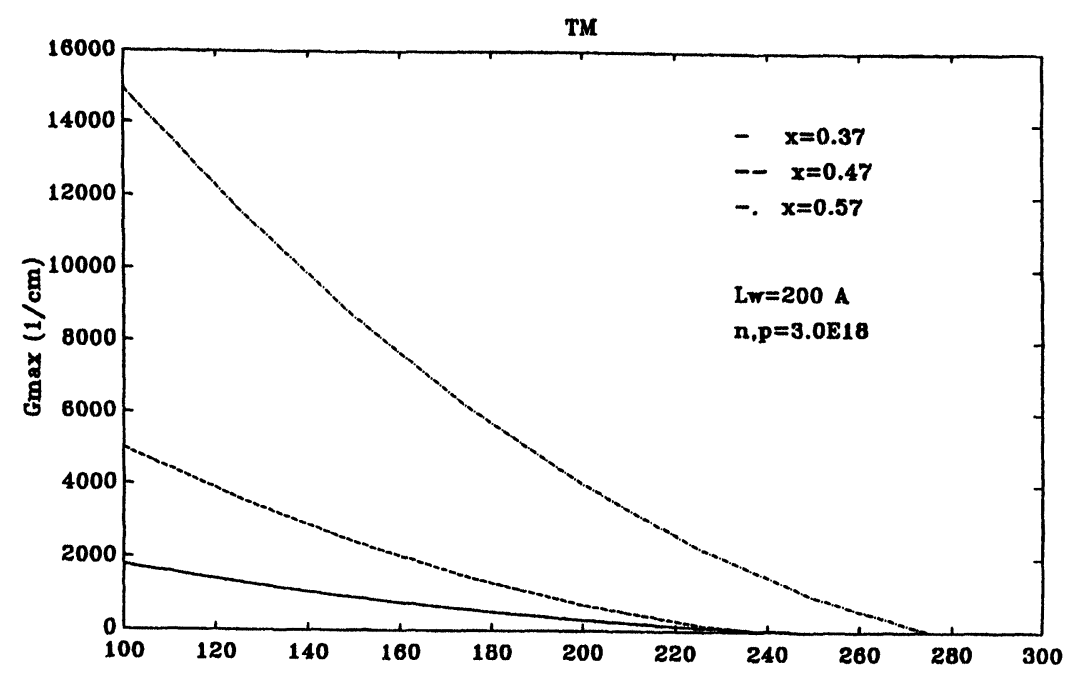

(b)

Temperature (K)

FIGURE 6 (Continued).

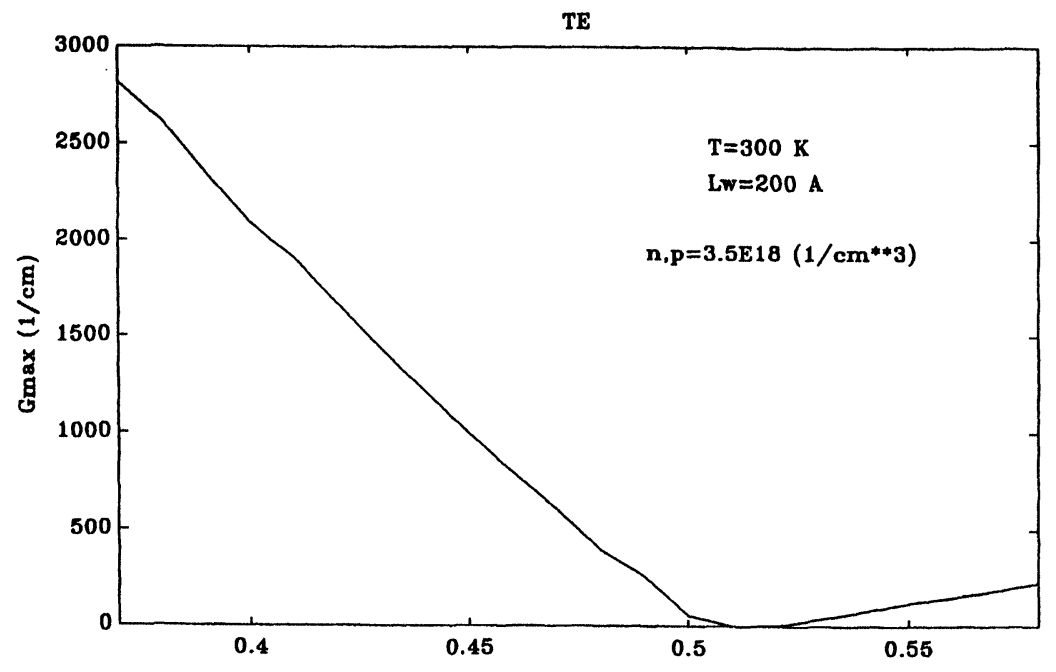

(a)

Ga mole fraction $x$

FIGURE 7 The (a) TE, (b) TM mode peak gain $v$ s. Ga mole fraction $x$. 


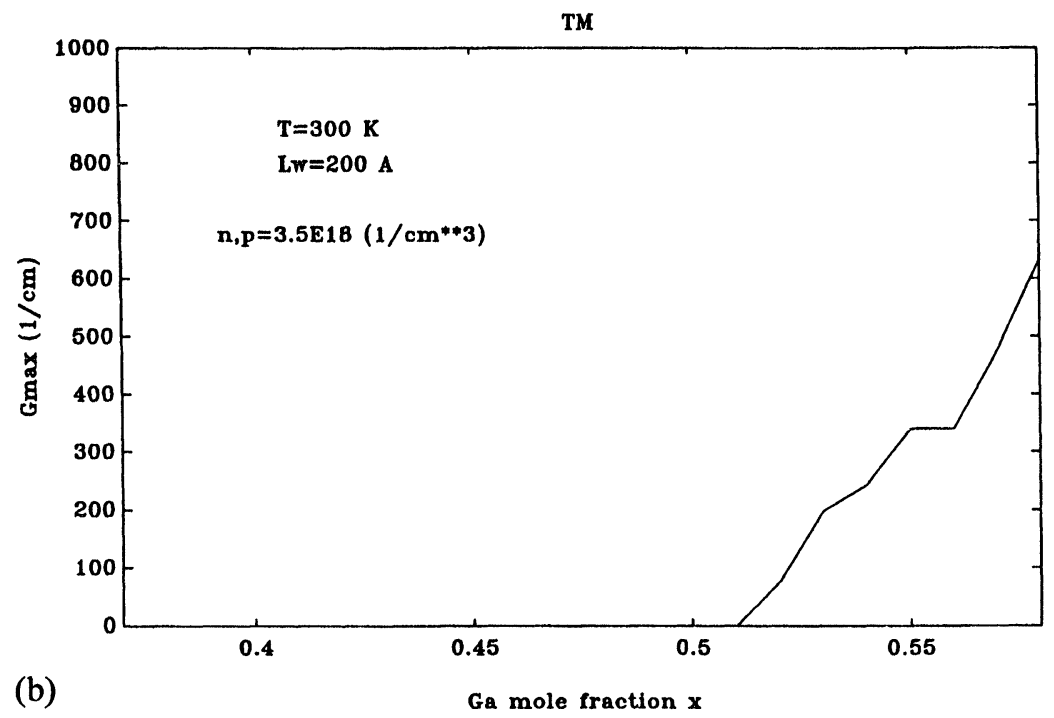

FIGURE 7 (Continued).

\section{CONCLUSIONS}

The effects of a biaxial strain on optical gain and refractive index change of a strained $I n_{1-x} G a_{x} A s_{y} P_{1-y} / I n_{1-x} G a_{x} A s$ quantum well lasers are studied theoretically using multiband effective mass equation, deformation potential theory and Fermi-Golden rule with broadening effect taken into account. The band structures are substantially altered by the biaxial strain. In particular, the inversion of LH1 and HH1 would change the dominant polarization of laser light from TE mode to TM mode. It is found that the appropriately biaxial strain would enhance the optical gain, thus will be benefited for reducing the threshold current. However, the biaxial strain would weakened the optical confinement and strengthened the temperature dependence of optical gain, these will be harmful to reduce the threshold current. It is also interesting to note that the minimum peak gain occurred when a small tensile strain is applied. The results emerging from our studies are helpful for understanding the guideline of designing strained-layer quantum well lasers. 


\section{References}

[1] Laidig, W. D., Caldwell, P. J., Lin, Y. F. and Peng, C. K. (1984). "Strained-layer quantum-well injection laser", Appl. Phys. Lett., 44, 653-655.

[2] Thijs, P. J. A. and Van Dongen, T. (1989). "High quantum efficiency, high power, modulation doped GaInAs strained-layer quantum well laser diodes emitting at $1.55 \mu \mathrm{m} "$, Electron. Lett., 25, 1735-1737.

[3] Koren, U., Oron, M., Young, M. G., Miller, B. I., De Miguel, J. L., Raybon, G. and Chien, M. (1990). "Low threshold highly efficient strained quantum well lasers at 1.5 micrometer wavelength", Electron. Lett., 26, 465-467.

[4] Williams, R. L., Dion, M., Chatenoud, F. and Dzurko, K. (1991). "Extremely low threshold current strained InGaAs/AlGaAs laser by molecular beam epitaxy", Appl. Phys. Lett., 58, 1816-1818.

[5] Osbourn, G. C. (1984). "InAsSb strained-layer superlattices for long wavelength detector applications", J. Vac. Sci. Technol. B., 2, 176-178.

[6] Zipperian, T. E. and Drummound, T. J. (1985). "Strained-quantum-well, modulation-doped, field-effect transistor", Electron. Lett., 21, 823-824.

[7] Rosenberg, J. J., Benlamri, M., Kirchner, P. K., Woodall, J. J. and Petit, G. D. (1985). "An $I n_{0.15} G a_{0.85} A s / G a A s$ pseudomorphic single quantum well HEMT", IEEE Device Res. Conf. Tech. Dig., EDL-6, pp. 491-493.

[8] Yablonovitch, E. and Kane, E. O. (1986). "Reduction of lasing threshold current density by the lowering of valence band effective mass", J. Lightwave Technol., 4, 504-506.

[9] Sanders, G. D. and Chang, Y. C. (1985). "Effects of uniaxial stress on the electronic and optical properties of $G a A s-A l_{x} G a_{1-x} A s$ quantum wells", Phys. Rev. B, 32, $4282-4285$.

[10] Ahn, D. and Chuang, S. L. (1988). "Optical gain in a strained-layer quantum-well laser", IEEE J. Quantum Electron., 24, 2400-2406.

[11] Yariv, A., "Quantum Electronics", Wiely, New York, 1974.

[12] Datta, S. (1989). "Quantum Phenomena", ADDISON-WESLEY, pp. 185-251.

[13] Luttinger, J. M. and Kohn, W. (1955). "Motion of electrons and holes in perturbated periodic field", Phys. Rev., 97, 869-883.

[14] Broido, D. A. and Sham, L. J. (1985). "Effective masses of hole at GaAs-AlGaAs heterojunctions", Phys. Rev. B., 31, 888-892.

[15] Asai, H. and Oe, K. (1983). "Energy band-gap shift with elastic strain in $G a_{x} I n_{1-x} P$ epitaxial layer on (001) GaAs substrates", J. Appl. Phys., 54, 2052-2056.

[16] Coon, D. D. and Karunasiri, R. P. G. (1984). "New mode of IR detection using quantum wells", Appl. Phys. Lett., 45, 649-651. 

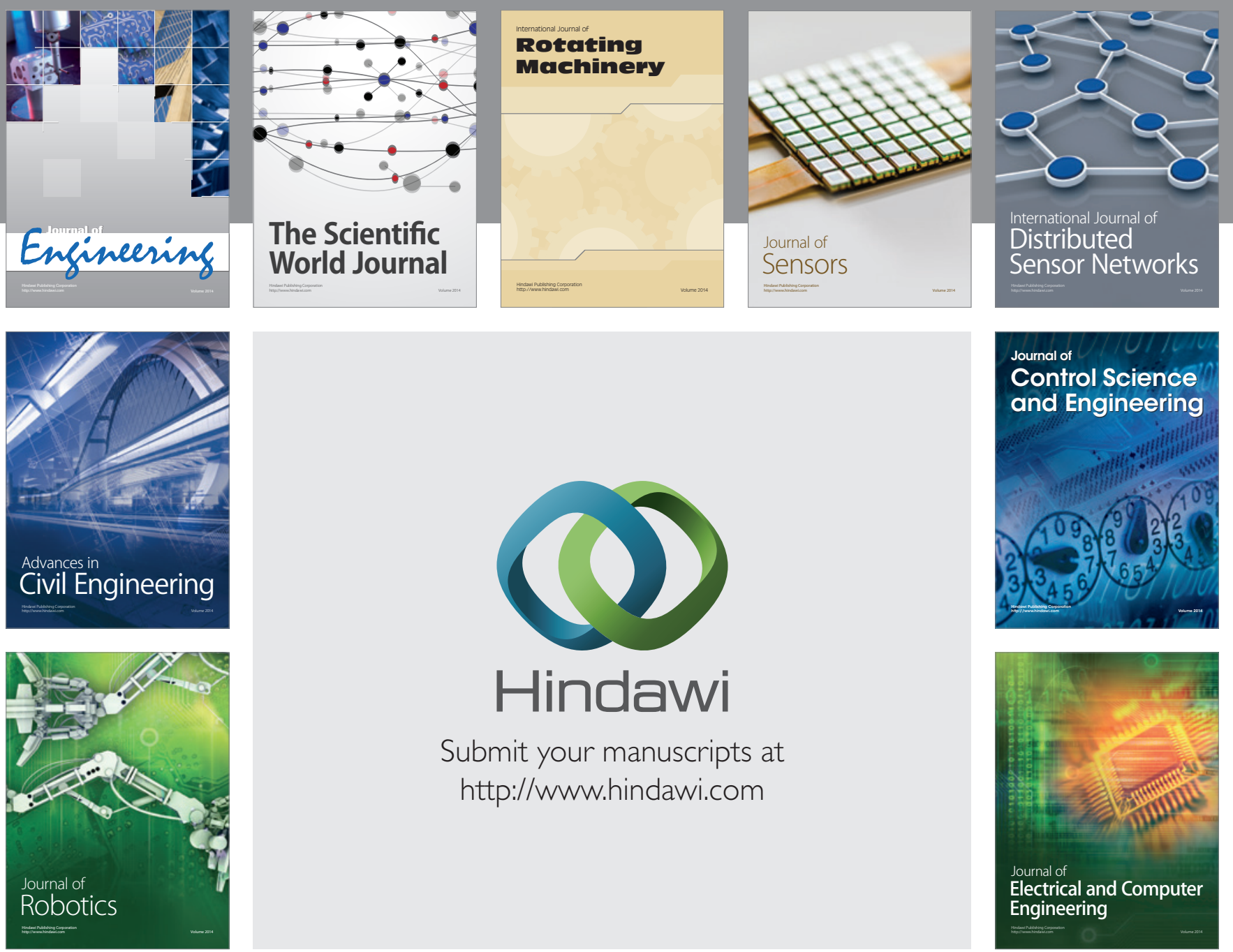

Submit your manuscripts at

http://www.hindawi.com
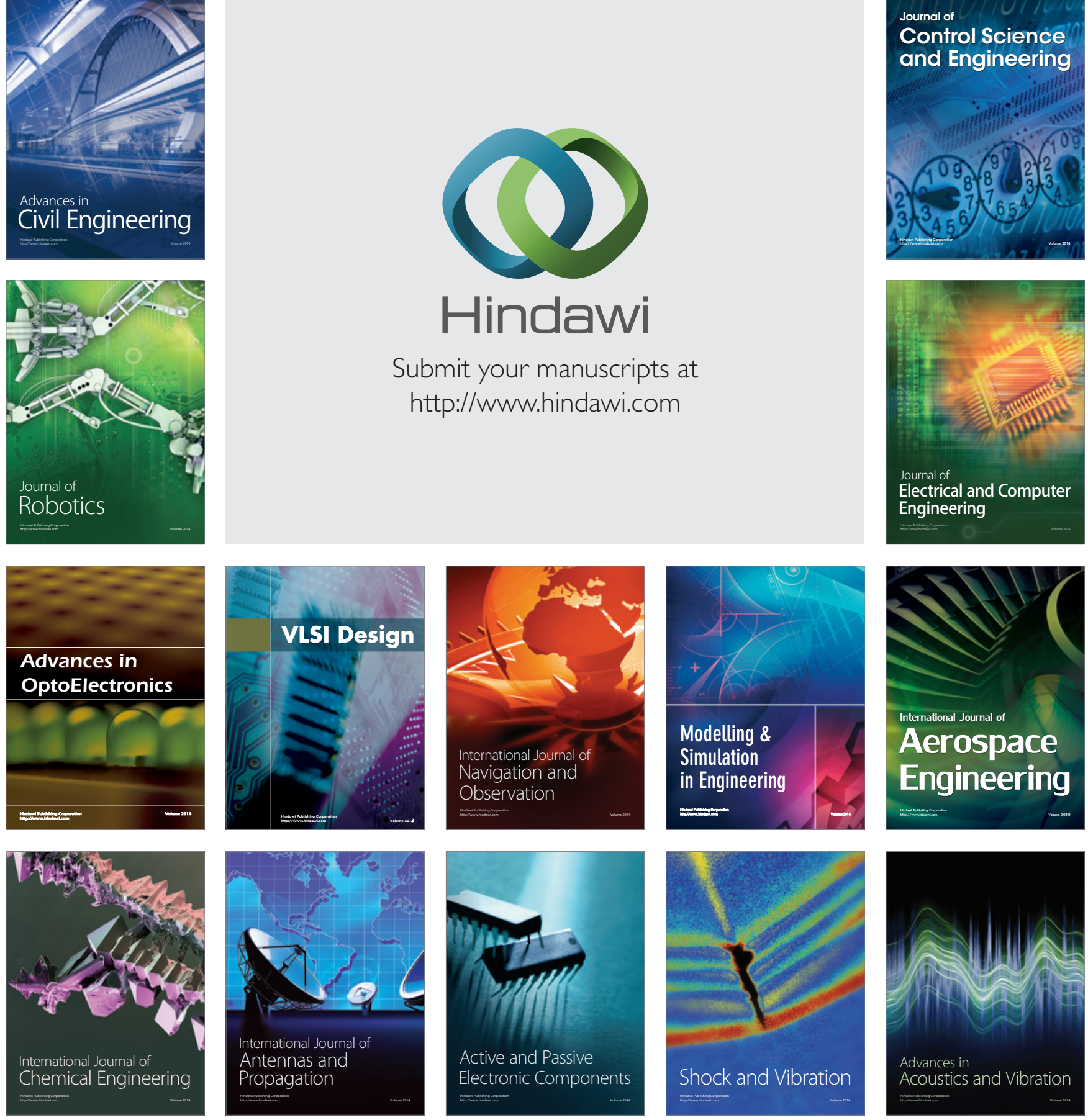